\title{
El Modernismo y el Nacionalismo decimonónico: dos expresiones de la modernidad
}

\author{
Luis Alejandro Arias Ramírez ${ }^{1}$ \\ Recibido el 9 de junio de 2017, aceptado el 27 de junio de 2017
}

\begin{abstract}
Resumen
Tanto el Modernismo como el movimiento nacionalista liberal posindependentista representaron, es sus respectivos periodos, formas distintas de percibir la realidad centroamericana. No obstante, ambas ideologías ofrecen una serie de elementos comunes que plantean una relación intrínseca entre ambos, así como la noción de que se puede trazar una línea clara entre las dos. Tanto los movimientos nacionalistas como la ideología modernista poseen cada uno una serie de rasgos ideológicos, así como diversas formas de manifestarse y ciertos objetivos concretos en su planteamiento. Comprender todos estos elementos será el primer paso para, posteriormente, analizar y estudiar la relación entre ambos movimientos, sus rasgos comunes y sus diferencias más generales.
\end{abstract}

\section{Palabras clave}

Modernismo, Nacionalismo, Centroamérica, ideología, realidad, similitudes, diferencias.

\begin{abstract}
Both the Modernist and the post-independence liberal nationalist movement represented, in their respective periods of time, two different ways for observe and understand the reality of Centro America. Therefore, both ideologies offer some common elements, these ones have a close relation between them; being able to clearly tie both ideologies through these general elements. The Nationalist movements and the Modernist have their own ideological settings, manifestations and objectives, unique in their own speeches. Understand all of these elements will be the first step for the study of the relationship between both ideologies, their common characteristics and their most important differences.
\end{abstract}

1. Bachiller en Enseñanza del español por la Universidad Nacional de Costa Rica. Universidad Federada San Judas Tadeo, Costa Rica. roninedain@gmail.com 
90 El Modernismo y el Nacionalismo decimonónico: dos expresiones de la modernidad. Luis Alejandro Arias Ramírez

\author{
Keywords \\ Modernist, Nationalist, Central America, ideology, reality, common charac- \\ teristics, differences
}

\title{
Introducción
}

El movimiento modernista en Hispanoamérica, y sobre todo en Centroamérica, supuso un momento y un fenómeno social y cultural de suma importancia para la época llegando a crear todo un ámbito literario.

Al ser un movimiento fundamentalmente artístico, se tiende a considerar como sus mayores muestras y repercusiones aquellas que sobresalen, principalmente, en el campo de la creación estética. La literatura, por ejemplo, se ha visto nutrida por los aportes del Modernismo y es de sobra conocido el papel de autores como Darío en el desarrollo de las letras centro e hispanoamericanas.

No obstante, esta constante exaltación de los logros estético-culturales del Modernismo suele provocar que se pierda de vista el impacto ideológico y social que este movimiento llegó a tener. Principalmente, es sencillo olvidar, ocupados en el estudio puramente artístico o biografista de Darío y demás figuras modernistas, que detrás del sistema estético modernista existió también un sistema ideológico que permeaba más allá de la sola producción artística.

El presente trabajo busca determinar de qué manera y en qué grado la consolidación de los estados nacionales centroamericanos durante el siglo XIX pudo influir en dicha ideología modernista, principalmente en lo que respecta a la repercusión que tuvieron los procesos constitutivos de la identidad de las recién consolidadas naciones por medio de la literatura.

Existen, a lo largo de este periodo, diversos movimientos de carácter culturalintelectual, marcados generalmente por la actividad académica (y, claro está, literaria) de muchos pensadores decimonónicos centroamericanos. Estos intelectuales, como José Cecilio del Valle o Francisco Morazán, plantearon un modelo de nación basado en ciertos procesos nacionalistas a lo largo del istmo, los cuales estuvieron ligados a las ideas y al contexto pos-independentista de la época. Es importante remarcar, dentro de estos procesos, el papel de las letras en la construcción de la nación y la identidad.

Se trata entonces de procesos ideológicos que buscaron definir, entre otras cosas, la literatura, la cultura, la política, el modelo de sociedad y, con ellas, las nuevas naciones centroamericanas. Por tanto, no se puede estudiar estos acontecimientos sin estudiar también las propuestas estético-ideológicas preponderantes en la realidad centroamericana circundante. 
¿Hasta qué punto pudieron los proyectos nacionalistas centroamericanos del siglo XIX influir en la posterior ideología y estética modernista? ¿Qué distancia puede verse entre las realidades nacionalista y modernista? ¿Qué repercusiones pudieron tener los proyectos de construcción de los estados en toda Centroamérica en el ideario modernista? Todas estas son las interrogantes que se pretenden estudiar a lo largo del presente trabajo.

Para lograr esto, se proponen los siguientes objetivos: En primer lugar, se buscará determinar la estructura ideológica de los proyectos nacionalistas centroamericanos a través del pensamiento de intelectuales de la época posindependentista, principalmente el caso de José Cecilio del Valle; por otra parte, también se pretende determinar la estructura ideológica del Modernismo como movimiento estético, social y cultural para tener una visión clara y concisa de la ideología involucrada en el desarrollo de este movimiento.

Una vez alcanzados estos dos objetivos, se procederá a comparar de manera general la propuesta ideológica del modernismo con la propuesta ideológica nacionalista centroamericana para determinar las principales similitudes y diferencias. Finalmente se comentarán las conclusiones obtenidas.

\section{Ideología Nacionalista liberal}

Resulta entonces necesario, en primer lugar, analizar ahora la construcción de la ideología nacionalista del siglo XIX. En primera instancia, es posible hablar del liberalismo como un elemento fundacional en el sistema ideológico-político de la época. En este sentido, Rafael Cuevas explica lo siguiente:

La construcción del nacionalismo y la nación moderna fue una faena a la que se abocaron los Estados latinoamericanos en la segunda mitad del siglo XIX. En ella tuvieron un papel protagónico grupos de intelectuales positivistas inscritos en el proyecto de los liberales los cuales, al decir de Benedict Anderson, se dieron a la tarea de "imaginarla", en el marco de la construcción y afianzamiento del desarrollo capitalista (Cuevas: p. 5)².

En este punto, es posible observar el carácter fundamental del liberalismo en la construcción de los estados y las identidades durante este periodo. A partir de esta base, se fundamentó la "invención” de los nuevos estados nacionales posteriores a la independencia. Para esto, los gobiernos se valieron de diversos elementos tales como la literatura y el discurso científico e historiográfico para elaborar una noción de identidad nacional y crear conceptos como "patria", "nación”, “estado", etc.

2. Cuevas, R. (s.f.). "El contexto nicaragüense", en Sandino y la intelectualidad costarricense. Nacionalismo antiimperialista en Nicaragua y Costa Rica (1927-1934). Obtenido de http://www. repositorio.una.ac.cr/bitstream/handle/11056/2894/recurso_982.pdf?sequence=1 
Un ejemplo claro de esta nueva noción de "naciones centroamericanas" y de los elementos fundamentales son los postulados ideológicos de diversos intelectuales decimonónicos, Tal es el caso de José Cecilio del Valle quién, a través del texto escrito, buscó configurar las características y las formas de la nueva realidad centroamericana del siglo XIX.

En el caso del primero, Teresa García Giráldez comenta, a propósito de la ideología que se trasluce en los escritos de este pensador:

\begin{abstract}
Además por el hecho de unir a la diversidad, la patria es también para Valle sinónimo de felicidad y libertad, en el sentido positivo y revolucionario de la Independencia; es autonomía y ruptura de los vínculos que frenan el progreso; es amor a la libertad y se, aplica patria la tierra de hombres libres y, por tanto, felices. La voluntad de querer una patria libre justifica la ruptura del vínculo centralizador del poder español y de cualquier otro "dominio imaginario" (García Giráldez, 2009: p. 28).
\end{abstract}

En este caso, persiste una idea de patria positivista y fundamentada en el liberalismo.

Un dato interesante, y por demás curioso, sobre el pensamiento nacionalista es su postura ante la importancia de los espacios intelectuales y del periodismo como elementos necesarios en las nuevas repúblicas. Como indica Sajid Alfredo Herrera Mena:

Pero el régimen civilista decimonónico fue construido asimismo por la opinión pública de los sectores ilustrados a través de las sociabilidades de los cafés, los clubes, las plazas públicas o las sociedades literarias en donde era palpable la incidencia de las publicaciones periódicas (Herrera Mena, 2008: p. 78) ${ }^{3}$

No es de extrañar que alguien como José Cecilio de Valle estuviera tan involucrado en el periodismo, hasta el punto de fundar y dirigir El amigo de la Patria.

\title{
Ideología Modernista
}

Como hemos mencionado, resulta necesario definir también algunos de los rasgos ideológicos principales del movimiento. En primer lugar, es importante señalar que el modernismo no constituyó sólo un movimiento artístico finisecular. En ese sentido, la gran mayoría de los estudios correspondientes a este movimiento muestran preferencia por la temática literaria y por destacar la importancia de sus autores en la historia de las letras americanas. 
Ante este respecto, Carlos Real señala lo siguiente:

Si como pienso, entonces, la filiación escolástica no es capaz de dar cuenta cabal de la propensión ideológica del lote modernista, no quedaría, no queda otra salida que la de repasar empíricamente los rasgos más relevantes por su potencialidad de significación ideológica que sus textos y sus conductas presentan. Sólo desde ahí, sostengo, es dable verificar las ambigüedades y latitudes que ambos repertorios de señales contienen; sólo desde ahí partir para un juicio de consistencia de lo que como "ideología" pudieran valer (Real de Azua, 1977: p.4) ${ }^{4}$.

Según esta perspectiva, el estudio del movimiento Modernista como fenómeno literario no es suficiente para determinar el papel político y social de sus principales figuras ni para delimitar la dimensión ideológica del movimiento. Con respecto a esta última, el autor plantea, según lo observado "empíricamente", las siguientes características de la ideología modernista: "Cosmopolitismo, idealismo "antieconomista", elitismo, religiosidad, hispanismo, latinoamericanismo, antiyankismo, compasión social, culto del héroe, han sido identificados con variables índices de frecuencias y densidades en las obras y los comportamientos de los más connotados modernistas." (Real de Azua, 1977, p.4) ${ }^{5}$.

Por otra parte, la ideología modernista no fue un elemento incidental, una repercusión secundaria del trabajo literario de sus figuras. Estos fueron siempre conscientes de su orientación ideológica y cuál era su base. A este respecto, Alberto Acereda explica lo siguiente: “Los modernistas hispanoamericanos sabían lo que estaban practicando y lo que era, en esencia, su actitud: una nueva visión del mundo que, al calor del ideario liberal decimonónico, implicaba un impulso reformista que traspasó los límites de la literatura y el arte" (Acereda, 2003: p. 767) $)^{6}$

La postura de Acereda concuerda con los postulados de Real de Azua, dado que muchas de las características dadas por este último coinciden con la tesis de la base liberal ofrecida en las líneas anteriores. Siguiendo esta línea de pensamiento, es posible construir un panorama de la ideología modernista basada en el liberalismo y marcada por el cosmopolitismo y el latinoamericanismo.

Por otra parte, de este análisis se desprende otra idea interesante para el desarrollo del presente trabajo: la base liberal de la ideología modernista fue adquirida en el contexto hispanoamericano decimonónico. En otras palabras, el liberalismo del modernismo no fue un producto de este que posteriormente

4. Real de Azua, C. (1977). El modernismo literario y las ideologías. Escritura. Teoría y crítica literarias, 41-75.

5. Ídem

6. Acereda, A. (2003). El antimodernismo. Sátira e ideología de un debate transatlántico. Hispania, 761-772. 
94 El Modernismo y el Nacionalismo decimonónico: dos expresiones de la modernidad. Luis Alejandro Arias Ramírez

influyera en las diversas realidades nacionales centroamericanas; sino que fue la materia prima que nutrió el ideario modernista.

\section{Modernidad desde dos perspectivas}

Si tomamos un elemento como la importancia de los espacios intelectuales y de la prensa escrita durante el periodo liberal nacionalista y lo comparamos con las posteriores tendencias modernistas como el valor de la crónica entre sus principales exponentes o el valor que estos le daban a los lugares "bohemios", bien podríamos hablar de coincidencias interesantes y hasta perfilar una cierta relación, no obstante, resulta necesario comparar otros factores para determinar la cercanía entre ambos movimientos.

Es destacable que en ambos casos estemos hablando de tendencias y formas de pensamiento en todos los campos: político, social cultural... es claro que el movimiento nacionalista tuvo una mayor tendencia política y el modernismo, un mayor impacto cultural. No obstante, es destacable como en ambos la literatura tuvo un especial impacto, ya fuera como medio de expresión política o artística.

Por otra parte, la relación a través del liberalismo es clara en ambos movimientos, según lo que se ha comentado anteriormente. Dado esto, consideramos más oportuno comentar otros aspectos menos evidentes. En este sentido, resalta el cosmopolitismo como un claro ejemplo. En el caso del modernismo, este resulta ser un elemento clave en la ideología del movimiento. El buscar la modernidad en otros ámbitos fuera del contexto centroamericano su siempre una preocupación de los modernistas, la cual sin embargo, también tiene su correspondiente en los nacionalistas.

Retomando el caso de José Cecilio del Valle, quien comenta:

La ilustración es el necesario de todas las naciones y de todas las clases he individuos de que se componen las naciones. No es feliz el hombre que no puede satisfacer las necesidades que sufre; no puede satisfacer las que siente si no tiene libertad para pensar, hablar, y hacer cuanto le convenga y no sea dañoso a otros" (Valle, 2008: p.163) ${ }^{7}$.

Podemos afirmar que el espíritu ilustrado del periodo pos-independentista puede entenderse como una cierta forma cosmopolita de ver el mundo, de buscar la construcción del estado en los postulados de la llustración francesa como posteriormente los modernistas lo harían en los maestros de la lírica galesa en pos de una nueva forma de arte.

7. Valle, J. C. (2008). Obra Escogida. Caracas: Ayacucho. 
Analizando esto en más detalle, es posible encontrar una diferencia crucial entre ambas ideologías. Estos dos movimientos buscaban la modernidad, tal como lo comentamos al hablar del cosmopolitismo. No obstante, la dirección de esta búsqueda parece ser la principal diferencia entre ambas propuestas.

En ese sentido, los nacionalistas pretendían construir la nación a partir de modelos externos, entendiendo este proceso como insertar la modernidad a la región para crear sus proyectos de estados centroamericanos. El modernismo por otra parte buscaba entender la modernidad fuera de Centroamérica; no como una forma de evadirse de la realidad, sino como una manera de entender la misma realidad, no ya para construirla, sino para comprenderla desde una perspectiva distinta.

\section{Conclusiones}

Entre las principales conclusiones de podemos extraer de nuestro análisis esta la presencia de elementos comunes en ambas ideologías, los cuales muestran una relación entre el movimiento nacionalista y el modernismo. El liberalismo, el cosmopolitismo e incluso el periodismo son elementos que favorecen la conexión entre ambos movimientos.

Aun con las diferencias claras, como la tendencia política nacionalista y la estética modernista o la direccionalidad de ambas visiones (hacia adentro para construir la realidad centroamericana o hacia afuera para entenderla); es posible concluir que los dos movimientos siguen una misma línea ideológica donde el modernismo actúa como una respuesta al contexto construido por los nacionalistas pos-independentista; lo cual, en última instancia, es un reflejo de la dinámica de la modernidad, cuya percepción e interpretación cambia a lo largo del tiempo. 


\section{Bibliografía}

Cuevas, R. (s.f.). 'El contexto nicaragüense', en Sandino y la intelectualidad costarricense. Nacionalismo antiimperialista en Nicaragua y Costa Rica (1927-1934). Obtenido dehttp: / / www.repositorio.una.ac.cr/bitstream/handle/11056/2894/ recurso_982.pdf?sequence $=1$

Herrera Mena, S., La formación de los estados-nacionales en la América, 19.05.2008. Obtenido de http://www.er-saguier.org/crisisyestado-nacion.org/archivo/ lecturas/El_estado.pdf

Real de Azua, C. (1977). 'El modernismo literario y las ideologías', en Escritura. Teoría y crítica literarias, Caracas, año 2, $n^{\circ} 3$, 1977. Obtenido de http:// www.autoresdeluruguay.uy/biblioteca/Carlos_Real_De_Azua/lib/exe/fetch. php?media=elmodernismoliterario.pdf

Acereda, A. (2003). El antimodernismo. Sátira e ideología de un debate transatlántico. Hispania, Vol. 86, No. 4, Diciembre de 2003). Obtenido de https://www.jstor. org/stable/20062924?seq=1\#page_scan_tab_contents761-772.

Valle, José Cecilio, (2008). Obra Escogida. Tipografía Nacional de Guatemala, Caracas, Vemezuela. 